\title{
Effect of red blood cells aggregation on scattering coefficient
}

\author{
A. Szołna-Chodór, A. Kempczyński, and B. Grzegorzewski* \\ Department of Biophysics, Collegium Medicum in Bydgoszcz, Nicolaus Copernicus University, \\ ul. Jagiellońska 13, 85-067 Bydgoszcz, Poland
}

Received November 15, 2011; accepted December 20, 2011; published December 31, 2011

\begin{abstract}
Red blood cells (RBCs) suspended in plasma aggregate This paper presents a method to determine the scattering coefficient of RBC suspensions at $632 \mathrm{~nm}$ wavelength. Blood was obtained from healthy donors. The intensity of the coherent component of scattered light from the suspension located in a wedge-shaped container was measured during $35 \mathrm{~min}$ at a sample thickness from 127 to $547 \mu \mathrm{m}$, a hematocrits 0.01 to 0.04 . It is shown that the scattering coefficient linearly decreases with time at the early stages of RBC aggregation. The time dependence of the coefficient becomes nonlinear when the early aggregation is completed. The duration of the early phase of RBC aggregation decreases as hematocrit increases. At very small hematocrits $(\mathrm{Ht} \leq 0.01)$ the scattering coefficient is independent of the thickness of the sample. The effect of the sample thickness on the scattering at higher hematocrits has been demonstrated
\end{abstract}

RBCs suspended in plasma form many different structures. The kinetics of the formation of the structures depends on, inter alia, the aggregation properties of the cells, hematocrit and the size of the sample. Knowledge of optical parameters of suspensions can be useful in the study of their kinetics. Especially, the scattering coefficient is a parameter that quantitatively describes the suspensions thus it can be useful as a standardization factor in hematology.

The RBCs suspended in plasma coalesce to form linear aggregates called rouleaux and branched structures. Recently it has been shown that the formation of a network is followed by the rouleaux formation at physiological hematocrit [1]. Next, three-dimensional RBC aggregates are formed and finally a deposit is formed as a result of sedimentation [2]. The rouleaux formation is most frequently studied among the processes described above. Many experimental techniques were adopted to study the aggregation process [3]. Microscopic techniques permit direct observation of formed structures [4-8]. A major advantage of the methods is that the parameters of aggregation kinetics can be obtained by simple visual inspection of aggregating RBCs. However, microscopic techniques are criticized because only very thin samples of suspensions can be investigated. Light scattering methods have been used in the study of RBC suspensions and the parameters of isolated cells are well recognized for normal and disease deformed cells [9-12].

*E-mail: grzego@cm.umk.pl
Also various experimental and theoretical approaches were used in the study of RBC aggregations [13-17].

In this letter, we demonstrate a novel method to determine the scattering coefficient of suspensions of aggregating RBCs. Light scattering of the suspensions results in a coherent and diffuse component at small hematocrit and relatively small sample thickness. The measurements of the intensity of a coherent component of scattered light were performed. The scattering coefficient of suspensions of individual cells and its time dependence are determined. The effect of sample thickness on the scattering is analyzed.

Blood from adult healthy donors was drawn into a tube containing $\mathrm{K}_{3}$ EDTA and used up to 10 hours. The RBCs were separated by centrifugation at $3000 \mathrm{rpm}$ for $5 \mathrm{~min}$ at $4^{\circ} \mathrm{C}$. RBCs were suspended in autologous plasma with a hematocrit of 0.01 up to 0.04 , then the suspensions were gently shaken and placed in a wedge-shaped container. The experiment was performed at room temperature $21^{\circ} \mathrm{C}$ $\pm 1^{\circ} \mathrm{C}$. The investigations were carried out according to the ethical guidelines laid down by the Bioethical Commission at the Ludwik Rydygier Collegium Medicum in Bydgoszcz of the Nicolaus Copernicus University.

The experimental setup is designed to measure the intensity of a coherent component of scattered light. An $\mathrm{He}-\mathrm{Ne}$ laser light beam at $632.8 \mathrm{~nm}$ wavelength was collimated. The circular aperture with a diameter of $3 \mathrm{~mm}$ determined the size of the incident beam. The power of the incident light beam was less than $0.01 \mathrm{~mW}$. The collimated light beam illuminated the sample placed in a wedge-shaped container whose thickness varied from 127 to $547 \mu \mathrm{m}$. To test the dependence of the intensity of a coherent component of scattered light on the thickness of the sample, a motorized stage was used. The container was horizontally moved. The coherent component of scattered light was separated using a lens and aperture placed at a distance equal to the focal length of the lens. The intensity of the coherent component was detected by a photomultiplier. The signal from the photomultiplier was directed to an amplifier. The measurement of a single sample lasts $35 \mathrm{~min}$ and the duration of a single scan of the sample was $41 \mathrm{~s}$. 
The coherent transmittance of a sample of RBC suspension has the form [18]:

$$
C=\exp \{-\mu d\}
$$

where $\mu$ is the attenuation coefficient of the suspension and $d$ is the thickness of the sample. The attenuation coefficient $\mu=\mu_{s}+\mu_{a}$, where $\mu_{s}$ is the scattering coefficient and $\mu_{a}$ is the absorption coefficient. For RBCs at the incident wavelength $632.8 \mathrm{~nm}$ the absorption coefficient is small compared to the scattering coefficient, thus in this case $\mu \approx \mu_{s}[9,10,12,17]$.

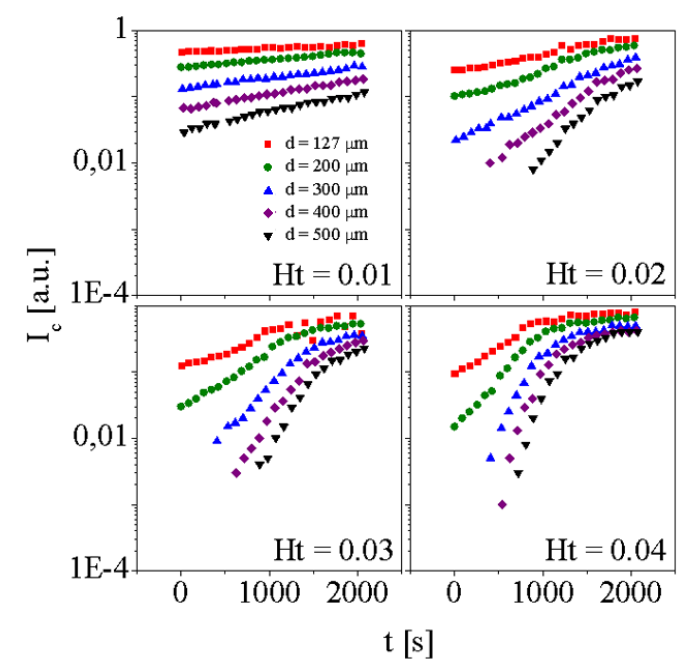

Fig.1. The intensity of coherent component $\mathrm{I}_{\mathrm{c}}$ of light scattered by RBC suspensions versus time at various hematocrits. The intensity is shown for sample thickness $d$ with values ranging from 127 to $500 \mu \mathrm{m}$ at every hematocrit.

The experimental data obtained from the samples are presented in Fig.1. The intensity of a coherent component of scattered light as a function of time is shown on a logarithmic scale. From this figure one can see that the scattering coefficient is a linear function of time at the early stages of the aggregation at every sample thickness. Later a departure from the linear dependence is observed.

The duration $T_{I}$ of the linear region of the coefficient versus time decreases as hematocrit increases. Figure 2 shows that the duration of the early stage of RBC aggregation significantly decreases with hematocrit of the suspension.

Figure 1 shows that the intensity of a coherent component of scattered light is a function of time, hematocrit and sample thickness. To describe this dependence an empirical formula for the intensity of a coherent component of scattered light was used.

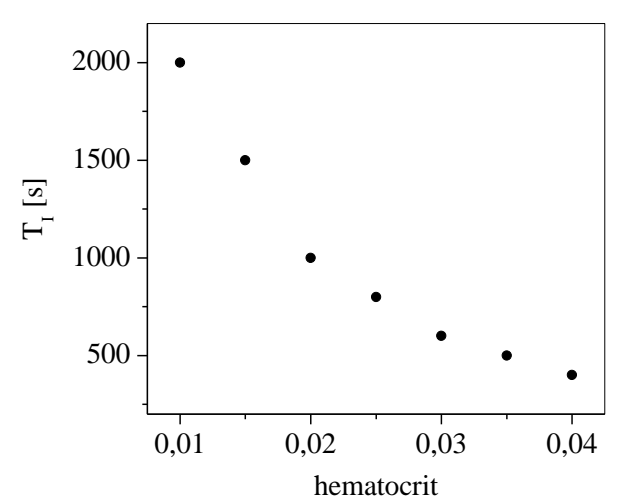

Fig. 2. Duration $T_{I}$ of the early stage of the RBC aggregation as a function of hematocrit.

We will show that the intensity of a coherent component of scattered light $I_{c}$ can be expressed by:

$$
I_{c}=I_{0} \exp \left\{-\left[\mu_{s} d-\left(A d+B d^{2}\right) t\right]\right\},
$$

where $\mu_{s}$ represents the scattering coefficients of individual RBCs occupying the container at $t=0$, parameters $A$ and $B$ describe the aggregation process and $I_{0}$ is the incident intensity.

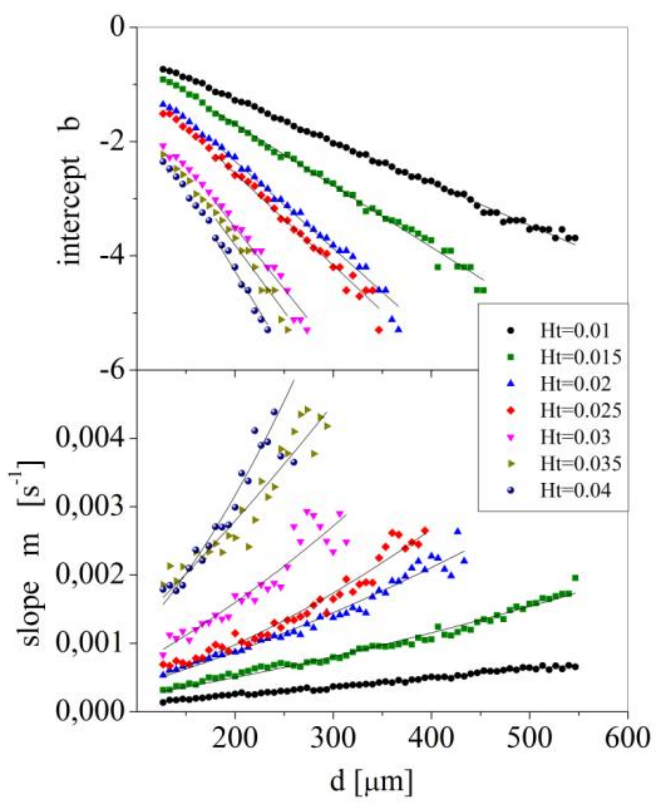

Fig. 3. Intercept $b$ and slope $m$ of the intensity logarithm versus sample thickness $d$ at hematocrit ranged from 0.01 to 0.04 . Points were taken from the experimental data and lines represents the best fits.

The logarithm of the intensity has the form:

$$
\ln I_{c}=\ln I_{0}-\mu_{s} d+\left(A d+B d^{2}\right) t=b+m t,
$$

where the intercept $b=\ln I_{0}-\mu_{s} d$ and the slope $m=A d+$ $B d^{2}$. 
In Fig. 3 the slope and intercept of the linear region of the intensity logarithm are shown as a function of sample thickness. From this figure one can see that the intercept $b$ is a linear function of thickness at a sample thickness from 180 to $540 \mu \mathrm{m}$. The slope $m$ is a nonlinear function of thickness and the departure from the linearity of the slope increases with an increase of the hematocrit.

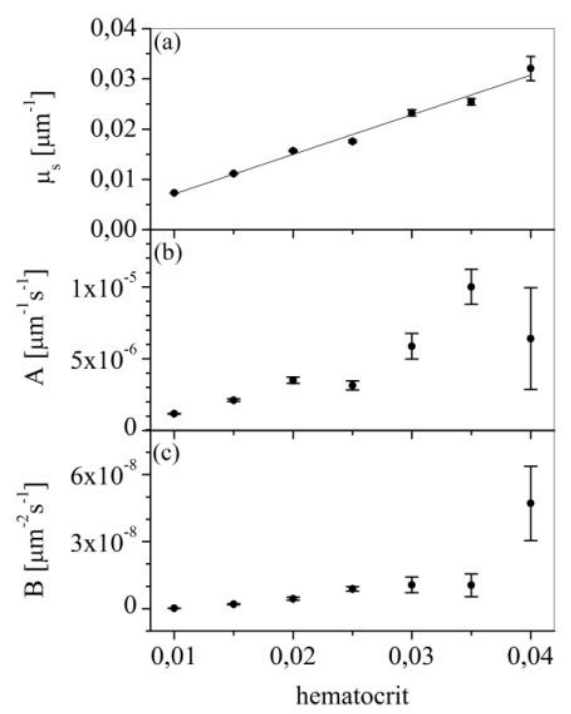

Fig. 4. Scattering coefficient $\mu_{s}$ (a), parameter $A$ (b) and $B$ (c) versus hematocrit. The solid line in (a) is a linear fit to the data.

The fits shown in Fig. 3 fit the data well. The scattering coefficient $\mu_{s}$ and parameters $A$ and $B$ extracted from the fits are shown in Fig.4. The hematocrit dependence of the scattering coefficient permits to find the scattering cross section of RBC. Since the volume of the cell is $90 \mu \mathrm{m}^{3}$, the cross section is $(69.4 \pm 1.4) \mu \mathrm{m}^{2}$. This value is close to that predicted by the Mie theory which is equal to $66.62 \mu \mathrm{m}^{2}$ [9]. The parameter $A$ increases with hematocrit. It is in agreement with the observations that the rate of rouleaux formation increases with hematocrit $[5,17]$. The parameter $B$ is very small at hematocrit 0.01 and then increases. However, it is difficult to interpret the meaning of the parameter from the preliminary results.

In conclusion, we have demonstrated a method of determining the scattering coefficient of RBC suspensions. Hematocrit and sample thickness dependence of the coefficient were taken into account. In general, the results show that the method can be useful in the study of both isolated RBCs and units that appear in the early stage of $\mathrm{RBC}$ aggregation. We have proposed an empirical formula to describe the time dependence of the intensity of the coherent component. This formula contains a quadratic term of sample thickness. Nonlinearity vanishes at hematocrit $\leq 0.01$, which greatly simplifies the interpretation of results. This method does not require a reference sample of the solution in which RBCs are suspended. Furthermore, the method has the ability to measure the sample thickness dependence of a coherent component intensity of scattered light using a single sample of RBC suspension. The advantages of the method make this approach very attractive for medical diagnostics.

\section{References}

[1] A. Pribush, D. Meyerstein, N. Meyerstein, Colloids and Surfaces B 75, 214 (2010).

[2] J. Mutrynowska, B. Grzegorzewski, Biorheology 44, 285 (2007).

[3] A. Pribush, N. Meyerstein, Recent Patents on Anti-Cancer Drug Discovery 2, 240 (2007).

[4] E. Ponder, Exp. Physiol. 16, 173 (1926).

[5] D. Kernick, A. W. L. Jay, S. Rowlads, and L. Skibo, Can. J. Physiol. Pharmacol. 51, 690 (1973).

[6] T. Shiga, K. Imaizumi, N. Maeda, K. Kon, Am. J. Physiol.. Heart Circ. Physiol. 245, H259 (1983).

[7] G. Barshtein, D. Wajnblum, S. Yedgar, Biophys. J. 78, 2470 (2000).

[8] I.Cicha, Y.Suzuki, N.Tateishi, N.Maeda, Am. J. Physiol Heart Circ. Physiol. 284, H2335 (2003).

[9] J. M. Steinke, A. P. Shepherd, Appl. Opt. 27, 4027 (1988).

[10] M. Hammer, D. Schweitzer, B. Michel, E. Thamm, A. Kolb, Appl. Opt. 37, 7410 (1998).

[11] O. Ergül, A. Arslan-Ergül, L Gürel, J. Biomed. Opt. 15, 045004 (2010)

[12] A. Roggan, M. Friebel, K. Dörschel, A. Hahn, G. Müller, J. Biomed. Opt. 4, 36 (1999)

[13] A.V. Priezzhev, O.M. Ryaboshapka, N.N. Firsov, I.V. Sirko, J. Biomed. Opt. 4, 76 (1999).

[14] M.J. Pearson, H.H. Lipowsky, Am. J. Physiol. Heart Circ. Physiol. 279, H1460 (2000)

[15] S.V. Tsinopoulos, E.J. Sellountos, D. Polyzos, Appl. Opt. 41, 1408 (2002)

[16] L.D. Shvartsman, I. Fine, IEEE Trans. Biomed. Eng. 50, 1026 (2003)

[17] C.V.L. Pop, S. Neamtu, J. Biomed. Opt. 13, 041308 (2008)

[18] V. Twersky, J. Opt. Soc. Am. 60, 1084 (1970) 